\title{
Senior High School Students' Perception on the Use of Masks to Prevent COVID-19 in Ghana
}

\author{
Emmanuel Aboagye $^{1^{*}[(])}$, Ishmael Owen Opoku², Nathanael Adu ${ }^{3}$ \\ ${ }^{1}$ Physical Education Department, Akrokerri College of Education, Akrokerri, Ghana \\ ${ }^{2}$ Physical Education and Sports Department, Konongo Odumase Senior High School, Konongo Odumase, Ghana \\ ${ }^{3}$ Physical Education Department, Mampong Technical College of Education, Mampong, Ghana \\ E-mail: aboagyeemmanuel13@yahoo.com
}

Received: 6 July 2021; Revised: 4 August 2021; Accepted: 4 August 2021

\begin{abstract}
The present study examines the perception of senior high school (SHS) students on the coronavirus and the use of masks to prevent the disease. Semi-structured interviews were employed as a form of data collection with purposively sampled twenty-four (24) senior high school students comprising twelve (12) males and twelve (12) females aged sixteen to nineteen (16-19) years. Thematic analysis was used in analysing the qualitative data gathered. Six (6) themes emerged that centered around SHS students' perception of the coronavirus, wearing of masks, health effects and other general ideas on the pandemic. The main themes were the mode of spread, symptoms, prevention, reasons for wearing masks, the health effects of wearing masks and other issues. The findings were discussed in line with recent literature and recommendations have been made to the stakeholders for policy direction. In sum, the students usually follow the safety protocols instituted by the World Health Organisation (WHO) and the government to stay alive and protected as they are aware of the adverse effects of contracting the disease which include death and liver damage when one even recovers.
\end{abstract}

Keywords: COVID-19, health effects, preventive measures, nose/face masks, oxygen

\section{Introduction and background}

The wearing of masks to control airborne infections is well researched in several pieces of literature (Tang et al., 2009; Liu \& Zhang, 2020; Chan \& Yuen, 2020; Humphreys, 2020; Guan et al., 2020). In their research, Tang et al. (2009) affirmed that human coughing moves at a faster speed into the surrounding atmosphere. However, wearing N95 masks traps the cough and prevents airborne transmission by blocking the droplets or redirecting them in a harmless direction. Liu and Zhang (2020) in their correspondence on face masks reported that one coronavirus patient traveling in china infected five people on a vehicle without a mask but infected none when a mask was worn. This explains the importance of wearing face masks in an enclosed space. Humphreys (2020) concluded that the use of face masks assists to protect several infections which spread through droplets. In contrast, Humphrey contends that the wearing of face masks should be within a short period to reduce the contraction of hypoxia.

Does this mean the wearing of masks has health implications? Since the outbreak of the COVID-19, there have been many attempts to better understand the origin of the virus, how it affects our health and how the use of masks to

Copyright (C2021 Emmanuel Aboagye, et al.

DOI: https://doi.org/10.37256/ser.2220211030

This is an open-access article distributed under a CC BY license

(Creative Commons Attribution 4.0 International License)

https://creativecommons.org/licenses/by/4.0/ 
prevent the spread can at the same time affect our health. There is limited information on the effectiveness of the use of face masks in filtering respiratory viruses and reducing viral release from an individual with respiratory infections (MacIntyre \& Chughtai, 2015). Researchers and scholars argue that the droplets from an infected person contain the virus when they cough, sneeze, talk, among the rest. Therefore, wearing masks prevents the virus from entering the nose or the mouth (Greenhalgh et al., 2020). Although the wearing of masks is very useful in preventing the contraction of the disease, the use of N95 masks for a long period can cause adjustments in the blood chemistry leading to changes in the level of consciousness if severe (Humphreys, 2020). Besides, despite the mask serving as a physical barrier to viruses, bacteria, etc., using it also blocks the openings of the nose and mouth, making breathing difficult. This may result in insufficient blood flow that provides adequate oxygenation leading to hypoxia and anoxia (Humphreys, 2020). Humphreys further reported that the condition is more likely to affect people with breathing difficulties, such as smokers, people suffering from obesity and individuals with Chronic Obstructive Pulmonary Disease (COPD) or emphysema.

Mo et al. (2020) applauds that wearing face masks is intolerable among people with COPD condition as this action will worsen their breathlessness. Wearing a mask can make an individual inhale a certain amount of the previously exhaled carbon dioxide into his or her respiratory system and this may cause other health risks. Research indicates that breathing in carbon dioxide can cause fast breathing, fast heart rate, emotional upset and tiredness (Zhang et al., 2020). Zhang et al. (2020) further argued that despite the use of masks to prevent viruses and bacteria from entering a person's respiratory system, the impacts on thermoregulation are small. They further advise that wearing masks in the heat or warm conditions or environment may make one feel warmer and sometimes uncomfortable. For instance, an exhaled air can enter a person's eyes which can make him touch the eyes with the hands and get infected if the hand is contaminated. Regarding this, homemade cloth masks or bandannas are more permeable and should be worn within a short time to avoid complications (Humphreys, 2020).

Studies by Humphreys (2020) and Zhang et al. (2020) confirm the usefulness of the wearing of the nose/face mask but inappropriate use of it may bring some ill-health problems to the wearer and his environment. According to Desai and Aronoff (2020), people should not touch their worn masks frequently and must change their single-used masks frequently or wash them regularly. Again, the researchers maintained that people should dispose of worn masks correctly and adopt other management measures, like washing of hands regularly with soap under running water, practicing social or physical distancing of at least one meter, in addition to other practices, to reduce the risks of contracting the virus. Furthermore, mass usage of disposable mask-wearing can cause increased littering in an environment which can lead to infectious hazards. Research conducted among the UK population shows that the number of masks to be disposed of in a day can result in environmental hazards because about 42,000 tonnes of potentially contaminated plastic waste cannot be recyclable in a year (Perski et al., 2020). Therefore, reusable masks are preferred.

Based on the background information and the review of related literature, the conceptual framework below was developed.

In May 2020, senior high school students in Ghana were sent back to school to commence with academic work. Most of the students who were in the final year were expected to prepare for the West Africa Senior School Certificate Examination. Personal Protective Equipment (PPEs) made up of masks and hand sanitizer were distributed to the students after an initial screening. Besides, students were enlightened on the safety protocols which make contracting the disease uncertain. However, the number of hours spent at school requires the student to wear a mask for at least four hours. Studies concerning the use of face masks to prevent contracting COVID-19 have focused on benefits and effects on the wearer without examining the perception of the users to determine how they feel about the use of the masks. Again, these studies did not focus on the perception of students or people in educational institutions who wear masks for a longer period to attend classes. Considering this, the present study purposely examined the perceptions of senior high school students on the coronavirus pandemic and the use of masks to control the pandemic. It is hoped that the findings from this study will not only inform the government about the awareness level of the SHS students on the COVID-19 pandemic and the observance of the safety protocols, but it will likewise inform mask manufacturers of the need to adjust their products to promote the health and well-being of the users. 


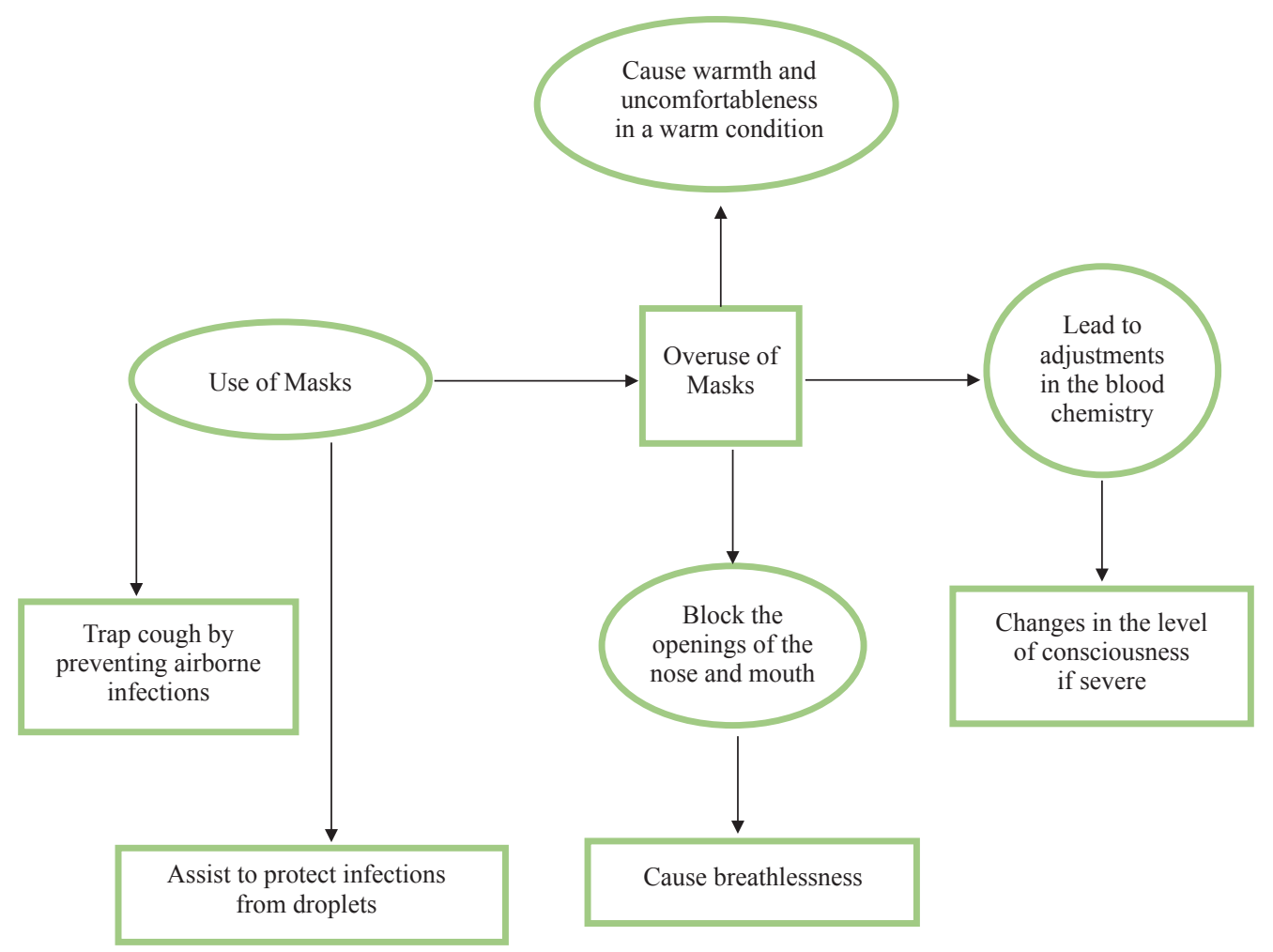

Figure 1. Conceptual framework on the perception of the use of masks to control the spread of Covid-19

From the above Figure 1, it could be deduced that the use of masks traps cough by preventing airborne infections and also, assists to protect infection from droplets. However, the overuse of masks can result in adverse effects such as causing warmth and making one feel uncomfortable in a warm condition, blocking the openings of the nose and mouth which can lead to breathlessness. Again, the overuse of masks can lead to adjustments in the blood chemistry causing changes in the level of consciousness if severe.

\section{Methodology}

\subsection{Research design}

A qualitative case study was used to describe students' perceptions of the coronavirus and the wearing of nose masks to stop the spread of the pandemic. In this regard, students' experiences with the wearing of nose masks were shared during the data collection process. Twenty-four senior high school students were purposively sampled to take part in a semi-structured interview which took place between June and July 2020. The participants were made up of twelve females and twelve males. A purposeful sampling technique was used to select the sample for the study. This was adjudged to be the best approach as the participants were chosen based on their willingness and readiness to give out the information needed by the researchers, and this approach assisted the researchers to get a piece of reliable information (Bernard, 2002).

To ensure reliability, five questions were observed throughout the collection of data (Egli et al., 2014): (a) Did the descriptions capture the experience? (b) Did the structure match the participant's experience? (c) Did the structure emerge from the data? (d) Did the theme and category titles relate to the primary source material? and (e) Do others see the description? Throughout data analysis, these questions were addressed by the researcher which led to the working and reworking of the thematic structure. Member checking, where transcripts were reviewed by the participants to ensure recordings as accurate to their experiences (Patton, 2002), was used to check the reliability of the results. 


\subsection{Instrument}

Before the main interviews, a pilot interview was conducted with one of the teacher's children who uses face masks to attend classes. The interview guide was developed based on the previous literature on COVID-19 and the use of masks. The interview guide contained predetermined schedules of open-ended and standardised questions. The relevance of the standard questions was to ensure that each interview suites individual differences (Patton, 1990), while the predetermined schedule was meant for consistency. The pilot interview assisted to make some relevant changes to the order and wording of the interview guide. The interview began with a general question to usher the participants into the study. For instance, the first question like "Describe what you know about the coronavirus pandemic?", was meant to introduce the students into the interview and prepare them for the next set of questions. Sentences like "could you tell me some more?" were used to gather more information from the participants on a particular question. The subsequent question was "what is the mode of the spread of the virus?" was asked to enable students to freely come out with their answers and avoid researchers' biases. Similar questions used to avoid biases in the interview were "what are some of the symptoms of the virus?", "what are some of the ways you can protect yourself from contracting the virus?". Questions relating to the use of masks to protect the virus started with "How can the wearing of the nose/face mask assist in the prevention of the spread of the virus?". This question was to let students mention ways that masks can assist stop the spread of the virus. The next set of questions that were used to determine the effects of the wearing of masks were asked in a manner devoid of biases. For example, "What are some of the challenges that you face when wearing the mask on campus?" and "How does the wearing of nose/face mask affect your health?". This allows the participants to mention some of the challenges they encounter when wearing masks on their own without any directions. The last question was to give the participants audience to mention other things about the virus that the researchers did not capture in the interview guide "Is there anything that you would like to say about the coronavirus?".

\subsection{Procedure}

Before the interviews, the purpose and ethics of the study were explained to the participants. Again, contacts of parents and guardians of the students who were less than 18 years were collected and the purpose of the study together with the ethical issues were explained in detail on the phone to the parents before the interviews were conducted for such students. Consent forms were given and signed by each student who participated in the interview. Participants were made to be aware that they can decide to stop at any time as participation is voluntary and that there is no monetary reward for participation. Again, participants were informed that the interviews were being recorded for accuracy. The interviews were conducted mainly after class and on weekends. Respondents' responses in the interview were recorded and transcribed verbatim. After the transcription, the recorded version of the interviews was played to the participants to attest whether they represent what they said for accuracy. Besides, the participants were assured of anonymity and confidentiality. These were done in line with ethical conditions. Pseudonyms were used to hide the identity of participants. The interviews lasted for about 35-40 minutes. The sessions were conducted in line with COVID-19 protocols which ensure that the distance between the interviewer and the interviewees was at least two meters.

\subsection{Data analysis}

The recordings from the interviews were transcribed verbatim and analysed thematically by following the steps of Braun and Clarke (2006). The analyses involved six phases: in the first stage, familiarizing with the data, the data were reread severally to ensure that the initial ideas were written down. At the second stage, the generation of early codes' interesting features of the data was coded across data set and was gathered based on the relevance to each code. During the probings for themes, the codes were organised into possible themes, and all data that were appropriate to each possible theme were collected. Afterward, the themes were reviewed by comparing them to the coded extracts and the entire data collected. In the fifth phase which involves defining and naming the themes, a clear definition and names of each theme were generated. And the last stage was producing the report. Here, the data were rechecked with the research questions and the literature to produce a scholarly report of the analysis. 


\section{Results}

Table 1. Summary of the results

\begin{tabular}{|c|c|c|c|}
\hline Number of participants & Themes & Codes & Frequency \\
\hline & & Getting in touch with infected people & 24 \\
\hline & & Touching infected surfaces & 12 \\
\hline \multirow[t]{4}{*}{24} & Mode of spread & Contact with droplets on infected people & 14 \\
\hline & & Transfer from human to humans & 24 \\
\hline & & Transfer from animals to humans & 10 \\
\hline & & Fever, dizziness & 19 \\
\hline \multirow[t]{3}{*}{22} & Symptoms & Coughing, sneezing & 34 \\
\hline & & Severe headache & 33 \\
\hline & & Washing hands regularly with soap under running water & 52 \\
\hline \multirow{3}{*}{24} & & Using about $70 \%$ alcohol made hand sanitizers & 45 \\
\hline & Prevention & Wearing face shield and nose masks & 31 \\
\hline & & Keeping a distance of about 2 meters & 26 \\
\hline \multirow{11}{*}{22} & \multirow{11}{*}{ Health effects of wearing masks } & Seizes carbon dioxide and reduces oxygen in the brain & 25 \\
\hline & & $\begin{array}{c}\text { Can cause death to people with respiratory } \\
\text { diseases-asthma }\end{array}$ & 15 \\
\hline & & Prevents the wearer from getting enough oxygen & 12 \\
\hline & & Difficulties in breathing & 54 \\
\hline & & Can lead to suffocation if worn for a longer period & 18 \\
\hline & & Can lead to cold & 27 \\
\hline & & Strains and pains around the ears & 20 \\
\hline & & Cannot hear colleagues talking at times & 40 \\
\hline & & Rashes around the face and ears & 12 \\
\hline & & Loss of smell and taste & 13 \\
\hline & & Headache & 48 \\
\hline \multirow{3}{*}{24} & \multirow{3}{*}{ Reasons for masks } & Protects infection from person to person & 40 \\
\hline & & $\begin{array}{l}\text { Prevents droplets of an infected person } \\
\text { from falling on you }\end{array}$ & 26 \\
\hline & & $\begin{array}{l}\text { Traps droplets of an infected person from getting } \\
\text { in touch with your nose and mouth }\end{array}$ & 12 \\
\hline \multirow{3}{*}{15} & & If you are cured your liver will get damaged & 53 \\
\hline & Other issues & $\begin{array}{l}\text { Adhering to the presidents' protocol is the best way } \\
\text { to protect from contracting the virus. }\end{array}$ & 28 \\
\hline & & The WHO protocols should be observed & 44 \\
\hline
\end{tabular}


The responses from the 24 students are provided in the form of themes and codes (Table 1). The responses were grouped based on the interview questions. Based on the questions, six (6) themes emerged that centered around SHS students' perception of the coronavirus, wearing of masks, health effects and other general ideas on the pandemic. The main themes are the mode of spread, symptoms, prevention, reasons for wearing masks, the health effects of wearing masks and other issues.

\subsection{Mode of spread}

This category involved five (5) codes: getting in contact with an infected person, touching infected surfaces, contact with droplets of an infected person, transfer from human to humans and transfer from animals to humans. All the students interviewed mentioned at least three factors that relate to the mode of spread of the coronavirus. In brief, this category provided knowledge on students' understanding of the means through which one can contract the coronavirus.

One participant stated:

"They say that when an infected person shakes your hand or talk and then the saliva settles on you, you are going to get the coronavirus".

Another participant also maintained:

"Apart from getting in contact with an infected person, you can also contract it when an infected person touches something and you also touch it, you can get the virus".

These statements revealed that students are aware of the means of contracting the virus.

\subsection{Symptoms}

Identifying symptoms of the coronavirus disease cannot be overlooked. Early detection of the disease enables one to seek the necessary assistance and to protect the virus from spreading further. The following codes were generated from the symptoms: fever, coughing, sneezing, severe headache and dizziness. Overall, this theme revealed the symptoms associated with contracting the coronavirus pandemic. Students are aware that having any of the symptoms implies that they have to go for testing to determine whether they have contracted the coronavirus or not. All the participants' response contains at least four of the codes under the symptoms.

A student was of the view that:

"Yes, I am told the disease is associated with coughing, high body temperature above the normal 37 degrees

Celsius".

Another participant posited:

"The disease is associated with dizziness, coughing and severe headache similar to that of a common cold that we normally get especially during the harmattan or the dry season".

\subsection{Prevention}

Since there is no known treatment for the coronavirus pandemic, preventive measures are considered effective for controlling the spread and contracting of the virus. The next theme that emerged from the interview was prevention with the following codes generated: Washing hands regularly with soap under running water, using about $70 \%$ alcohol made hand sanitizers, wearing face shields and nose masks and keeping a distance of about two meters away from each other. All the participants mentioned almost all the codes under this theme which indicates that the students are abreast of the preventive measures put in place to control the spread.

A participant echoed:

"Wearing of a nose mask, using at least 70\% alcohol-based sanitizers, washing of hands regularly with soap under running water, avoiding crowding and practicing social distancing of 2 meters can help prevent contracting the coronavirus pandemic".

Another student supported the above statement with a response like:

"You have to wear your nose mask, distancing yourself from people of about 1 meter, washing your hands frequently under running water with soap for about 20 seconds, using alcohol-based hand sanitizers as often as possible". 
The most important revelation under this theme was made by a student to support a proposal by the WHO. "The WHO has brought some precautionary measures such as wearing of a nose mask, using alcohol-based hand sanitizers frequently, washing our hands under running water with soap and social distancing of about 1 meter".

\subsection{Reasons for wearing masks}

Three reasons were mentioned by the participants which formed the codes under this theme. They were given to protect infection from person to person, prevents droplets of an infected person from falling on you and traps droplets of an infected person from getting in touch with your mouth and nose. These formed the basic reasons why the wearing of masks has been considered a necessity especially when people are in a car or enclosed space like the classroom.

A participant revealed:

"The virus can spread through the spit of an infected person when talking. So, the mask can accumulate the virus in the spit and prevent it from entering the mouth or the nose of a person".

A student contends:

"If you wear the nose mask, if someone has the virus, he/she cannot infect you with the virus".

\subsection{Health effects of wearing masks}

This theme was considered because many people have expressed the uneasiness they encounter when wearing masks. The students' challenges in wearing the nose masks were of relevance in the present study as they spend more than four hours a day attending classes with the nose masks on. The codes that emerged were: Seizes carbon dioxide and reduces oxygen in the brain, can cause death to people with respiratory diseases-asthma, prevents the wearer from getting enough oxygen, difficulties in breathing, can leads to suffocation if worn for a longer period, can lead to cold, can lead to strains and pains around the ears, difficulties in hearing your colleagues when they talk at times, rashes around the face and ear and loss of smell and taste. At least four out of the ten codes were mentioned by the participants in the study.

A participant explains:

"It is not an easy task at all. Wearing it all day (Classroom for about 5-6 hours, dining hall, preps, etc.) in school as well as the dormitory with the same nose mask. This makes us get diseases such as cold which makes learning very uncomfortable and difficult. Notwithstanding, we also develop headaches and difficulty in breathing and this makes studies unbearable. Consequently, one worrisome thing is that, when someone develops the cold, headache and other kinds of stuff, the students distance themselves from you thinking that you have contracted the coronavirus".

With breathing difficulties, a student supports, "Ok, sometimes if I have not run or engaged in any vigorous physical activity, then I am ok with it. But when I start running then it seems I will start panting like a lion which makes me feel very uncomfortable". Apart from making breathing difficult, most of the participants agreed that there is a shortage in oxygen supply if one wears the mask as there are not enough holes in the materials used in making the masks.

"Ok wearing of the nose masks, if you are maybe suffering from any strange sickness that you need a lot of oxygen, wearing of the nose masks, is going to prevent you from getting the oxygen that you deserve since the holes in it are not enough for the oxygen to pass through, so someone might die as a result of it".

This statement was made by one of the participants to support the above argument.

\subsection{Other issues}

To determine whether students are contemplating doing away with the masks to avoid facing any of the challenges, it emerged from the responses that they will keep on wearing the masks despite the health implications. Students were afraid of contracting the virus and even the side effects after recovering from the pandemic. They were willing to obey the preventive measures by the $\mathrm{WHO}$ and the president.

A participant extols:

"Coronavirus they say is very deadly, if care is not taking and one gets that virus then definitely the person 
is going to die or if you don't die after you have been cured, it is going to cause damage to your liver, then damage of the liver too is a big problem since the liver performs a lot of functions to us as human beings, so if our liver gets damaged, then we might die, we might not survive for long".

\section{Discussion of findings}

The purpose of the study was to examine the perceptions of SHS students on the coronavirus and the use of masks to prevent the pandemic. The findings revealed that students are aware of the mode of spread of the virus, the symptoms associated with contracting the virus, prevention of the virus, reasons for wearing masks, the health effects of wearing masks and other relevant issues on the pandemic. The findings have exposed the decision by students to observe the WHO preventive measures and the government protocols despite several health repercussions associated with the use of masks. Students further argued that after recovering from the virus, there are other severe consequences including liver damage which affects the overall health of the person.

With regards to in-depth knowledge of the coronavirus pandemic, it was identified from the study that students are aware of the mode of spread of the virus, symptoms associated with contracting the virus and measures put in place to control the spread. Governments and the WHO have put up stringent measures to ensure that much information about the coronavirus is preached to everyone. Therefore, students observe common preventive protocols like washing hands regularly with soap under running water for at least thirty seconds, using at least $70 \%$ alcohol-based hand sanitizers as often as possible, wearing a face shield and nose masks and keeping a distance of about two meters. This somewhat supports the WHO COVID-19 transmission and preventive measures, cleaning one hand often, coughing or sneezing in bent elbow-not your hands, wearing a mask, avoiding touching of eyes, nose, and mouth, limiting social gatherings and time spent in crowded places, avoiding close contact with someone who is sick and cleaning and disinfecting frequently touched objects and surfaces (WHO, 2020).

Reasons for wearing masks produce codes such as preventing infection from person to person, proventing droplets of an infected person from falling on you and traping droplets of an infected person from getting in touch with your nose and mouth. For instance, Humphreys (2020) examined the importance of wearing masks in curtailing the COVID-19 pandemic and concluded that face masks help to protect against several respiratory infections that spread through droplets. Tang et al. (2009), confirms that wearing N95 masks traps the cough and prevents airborne transmission by blocking the droplets or redirecting them in a harmless direction. Liu and Zhang (2020) accentuate the importance of wearing masks in an enclosed space when he reported that one coronavirus patient traveling in China infected five people on a vehicle without a mask but infected none when he wears a face. Students should prioritise the wearing of masks to prevent infections as they are always in confined places, the classrooms, halls and dormitories.

Despite the numerous benefits of wearing masks, students expressed the health effects of their usage. Common among them include breathing challenges, headaches, colds and difficulties in hearing. The health challenges of wearing masks were identified by the same researchers that recommended the benefits of using them. Humphreys (2020) asserted that the use of N95 masks for a prolonged period may have alterations in the blood chemistry that could lead to changes in the level of consciousness if severe. Humphrey again observed that wearing masks for a longer period causes insufficient blood flow that provides adequate oxygenation (Ischemia) leading to hypoxia and anoxia. The condition is more likely to happen to those who are already predisposed to breathing difficulties, such as smokers, obese people, or individuals with Chronic Obstructive Pulmonary Disease (COPD) or emphysema (Humphreys, 2020). The above assertion was supported by Mo et al. (2020) who opined that wearing face masks should not be encouraged among people with COPD conditions as they worsen their breathlessness.

Inhaling one's carbon dioxide was identified as a challenge to the use of masks by the students. Students maintained that they inhaled carbon dioxide instead of oxygen because the masks prevent the carbon dioxide from escaping to allow oxygen. This was mentioned by Zhang et al. (2020) who indicated that inhaling carbon dioxide can cause rapid breathing, rapid heart rate, emotional upset and fatigue. Students admit to hearing difficulties when they wear masks and strains around the ears. This could be attributed to the nature of the weather as the heat can cause the areas around the ears, mouth and nose to sweat creating some discomforts. Zhang et al. (2020) indicated that wearing masks in heat or warm conditions or environments may make one feel warmer and sometimes uncomfortable. For instance, an exhaled 
air can enter into a person's eyes which can make him touch the eyes with the hands and get infected if the hand is contaminated.

The present study highlighted the challenges students in senior high schools encounter when wearing masks on campus. Despite the well-established significance of masks to prevent the COVID-19, it poses a threat to people with respiratory problems. Considering this, it will be relevant for people with respiratory challenges to be given special considerations on how to use masks. For example, people with asthma should be given a seating preference-sitting about two meters from the rest of the class so that they can remove the mask after some time when they are feeling uneasy. Again, people with severe respiratory infection can be supported with online learning if the authorities identify that such students cannot cope with the wearing of nose masks. Practicing physical distancing in classrooms can enable students to wear masks for a longer period. When students are far from each other in classrooms they can easily remove the masks at a point in time to reduce the risks associated with using masks for a longer period. Another alternative is for the school authorities to restructure the timetables in this pandemic era. Instead of spending about four hours before break sessions, schools can have a break after every hour to enable students to remove the masks for fresh air. The government should employ the services of more teachers so that large class sizes can be divided to enable students to attend classes in batching. In so doing, physical distancing can be promoted to limit the use of nose masks.

\section{Limitations of the study}

Ideally, twenty-four students from a single senior high school are considered a limitation as it does not allow the results to be extrapolated. Notwithstanding, in using a qualitative approach, few responses are considered reliable since the intent is to provide an in-depth account of individual responses on the topic under study. The respondents were students randomly selected to express their views on the topic under investigation and therefore, offered divergent views. Also, this study is a foundation for researchers to use clinical equipment to examine the major health risks of wearing masks for a long period.

Future studies should involve more students selected from different educational settings using a quantitative approach in a longitudinal study to identify the other health effects of using masks that were not captured in the current study.

\section{References}

Bernard, H. R. (2002). Research Methods in Anthropology: Qualitative and Quantitative Methods (3rd ed.). Alta Mira Press, Walnut Creek, California. http://ndl.ethernet.edu.et/bitstream/123456789/40820/1/17.pdf.pdf

Braun, V., \& Clarke, V. (2006). Using thematic analysis in psychology. Qualitative Research in Psychology, 3(2), 77101. https://www.tandfonline.com/doi/abs/10.1191/1478088706qp063oa

Chan, K. H., \& Yuen, K. Y. (2020). COVID-19 epidemic: Disentangling the re-emerging controversy about medical facemasks from an epidemiological perspective. International Journal of Epidemiology, 49(4), 1063-1066. https:// doi.org/10.1093/ije/dyaa044

Desai, A. N., \& Aronoff, D. M. (2020). Masks and coronavirus disease 2019 (COVID-19). Jama, 323(20), 2103. https:// doi.org/10.1001/jama.2020.6437

Egli, T., Czech, D. R., Todd, S. Y., Shaver, G. W., Gentner, N., \& Biber, D. D. (2014). The experience of Christian Prayer in Coaching: A qualitative investigation. Journal of Psychology and Christianity, 33(1), 45-57. https:// scholar.google.com/scholar?cluster $=14106632322556632269 \& h l=e n \& a s \_s d t=0,5$

Greenhalgh, T., Schmid, M. B., Czypionka, T., Bassler, D., \& Gruer, L. (2020). Face masks for the public during the covid-19 crisis. Bmj, 369, m1435. https://doi.org/10.1136/bmj.m1435

Guan, L., Zhou, L., Zhang, J., Peng, W., \& Chen, R. (2020). More awareness is needed for severe acute respiratory syndrome coronavirus 2019 transmission through exhaled air during non-invasive respiratory support: Experience from China. European Respiratory Journal, 55(3), 2000352. https://doi.org/10.1183/13993003.00352-2020

Humphreys, J. (2020). The importance of wearing masks in curtailing the COVID-19 pandemic. Journal of Family Medicine and Primary Care, 9(6), 2606-2607. https://pesquisa.bvsalud.org/global-literature-on-novel-coronavirus2019-ncov/resource/en/covidwho-801659 
Liu, X., \& Zhang, S. (2020). COVID-19: Face masks and human-to-human transmission. Influenza and Other Respiratory Viruses, 14(4), 472-473. https://doi.org/10.1111/irv.12740

MacIntyre, C. R., \& Chughtai, A. A. (2015). Facemasks for the prevention of infection in healthcare and community settings. British Medical Journal, 350, h694. https://doi.org/10.1136/bmj.h694

Mo, Y., Wei, D., Mai, Q., Chen, C., Yu, H., Jiang, C., \& Tan, X. (2020). Risk and impact of using mask on COPD patients with acute exacerbation during the COVID-19 outbreak: a retrospective study. Research Square; 2020. https://doi.org/10.21203/rs.3.rs-39747/v1

Patton, M. Q. (1990). Qualitative Evaluation and Research Methods (2nd ed). Newbury Park, CA: Sage. https://psycnet. apa.org/record/1990-97369-000

Patton, M. Q. (2002). Qualitative Research and Evaluation Methods. Thousand Oaks, CA: Sage.

Perski, O., Simons, D., West, R., \& Michie, S. (2020). Face masks to prevent community transmission of viral respiratory infections: A rapid evidence review using Bayesian analysis. Qeios. https://doi.org/10.32388/1SC5L4

Tang, J. W., Liebner, T. J., Craven, B. A., \& Settles, G. S. (2009). A schlieren optical study of the human cough with and without wearing masks for aerosol infection control. Journal of the Royal Society Interface, 6(6), S727-S736. https://doi.org/10.1098/rsif.2009.0295.focus

World Health Organization. (2020, April 2nd). Coronavirus disease 2019 (COVID-19): situation report, 73. https:// www.who.int/emergencies/diseases/novel-coronavirus-2019/situation-reports

Zhang, Y., Marshall, G., Beresh, S. J., Richardson, D., \& Casper, K. M. (2020). Multi-line FLEET by imaging periodic masks. Optics Letters, 45(14), 3949-3952. https://doi.org/10.1364/OL.392779 